\title{
Forkhead Protein FoxO1 Acts as a Repressor to Inhibit Cell Differentiation in Human Fetal Pancreatic Progenitor Cells
}

\author{
Zongzhe Jiang, ${ }^{1}$ Jingjing Tian, ${ }^{1}$ Wenjian Zhang, ${ }^{2}$ Hao Yan, ${ }^{1}$ Liping Liu, ${ }^{3}$ Zhenhe Huang, \\ Jinning Lou, ${ }^{2}$ and Xiaosong $\mathrm{Ma}^{1}$ \\ ${ }^{1}$ Shenzhen University Diabetes Institute, Shenzhen University, Shenzhen 518060, China \\ ${ }^{2}$ Institute of Clinical Medical Sciences, China-Japan Friendship Hospital, Beijing 100029, China \\ ${ }^{3}$ Shenzhen Hightide Biopharmaceutical Ltd., Shenzhen 518000, China \\ ${ }^{4}$ Department of Aging Medicine, The Sixth Hospital of Shenzhen Municipality, Shenzhen 518060, China
}

Correspondence should be addressed to Xiaosong Ma; xsma@szu.edu.cn

Received 13 October 2016; Revised 27 December 2016; Accepted 29 January 2017; Published 28 February 2017

Academic Editor: Andrea Tura

Copyright (c) 2017 Zongzhe Jiang et al. This is an open access article distributed under the Creative Commons Attribution License, which permits unrestricted use, distribution, and reproduction in any medium, provided the original work is properly cited.

\begin{abstract}
Our colleagues have reported previously that human pancreatic progenitor cells can readily differentiate into insulin-containing cells. Particularly, transplantation of these cell clusters upon in vitro induction for 3-4 w partially restores hyperglycemia in diabetic nude mice. In this study, we used human fetal pancreatic progenitor cells to identify the forkhead protein FoxOl as the key regulator for cell differentiation. Thus, induction of human fetal pancreatic progenitor cells for 1 week led to increase of the pancreatic $\beta$ cell markers such as Ngn3, but decrease of stem cell markers including Oct4, Nanog, and CK19. Of note, FoxO1 knockdown or FoxO1 inhibitor significantly upregulated Ngn3 and insulin as well as the markers such as Glut2, Kir6.2, SUR1, and VDCC, which are designated for mature $\beta$ cells. On the contrary, overexpression of FoxO1 suppressed the induction and reduced expression of these $\beta$ cell markers. Taken together, these results suggest that FoxO1 may act as a repressor to inhibit cell differentiation in human fetal pancreatic progenitor cells.
\end{abstract}

\section{Introduction}

Decrease of $\beta$ cell mass plays a crucial role in development of type 2 diabetes mellitus. Islet transplantation is a promising strategy to reestablish the $\beta$ cell mass; however, its usage is limited by the shortage of available islets [1]. Human fetal pancreatic stem cells have been found as a good source of insulin producing cells, given its capability of readily self-renewal and differentiating into insulin producing cells in vitro by differentiation at conditions resembling those of physiological environments [2]. Our colleagues have reported previously that these differentiated cell clusters generated from human fetal pancreatic progenitor cells exhibited more insulin contents and improved secretary capability and glucose response [3]. Transplantation of these cell clusters normalized hyperglycemia in diabetic nude mice [3]. Nevertheless, the key molecular in controlling differentiation of the human fetal pancreatic progenitor cells is still unknown.

It has been found that the forkhead transcription factor FoxOl is a prominent mediator in controlling pancreatic $\beta$ cell mass [4]. FoxO1 is most abundant isoform among FOXO class members in the adult pancreas and preferentially expressed in pancreatic $\beta$ cells, where it plays an essential role in $\beta$ cell growth and proliferation $[5,6]$. During mouse pancreatic organogenesis, FoxO1 is found in the pancreatic epithelium between e9.5 and 14.5 [7] and is implicated in pancreatic organogenesis [7]. Previous studies revealed that FoxO1 ablation in mice resulted in increase of juxtaductal $\beta$ cells [8] and insulin-positive cells generated from the gut epithelial cells [9]. Moreover, FoxO1 knockdown rescued the diabetic phenotype in insulin-resistant mice [10], whereas constitutive activation of FoxO1 caused hyperglyceridemia and impaired insulin secretion [11]. However, little is known 
of its role in regulation of $\beta$ cell development in the human fetal pancreas. In this study, we used human fetal pancreatic progenitor cells to identify the role of FoxO1 in cell differentiation.

\section{Materials and Methods}

2.1. Culture of Human Fetal Pancreatic Progenitor Cells. The present study was approved by the Clinical Research Ethics Committee of both Shenzhen University and ChinaJapan Friendship Hospital and conducted according to the principles of the Declaration of Helsinki. The human fetal pancreatic progenitor cells used for expansion were cultured in a $37^{\circ} \mathrm{C}, 5 \% \mathrm{CO}_{2}$ incubator in DMEM/F12 medium containing $5 \%$ fetal bovine serum, $40 \mu \mathrm{g} / \mathrm{L}$ leukemia inhibitor factor (LIF), $10 \mu \mathrm{g} / \mathrm{L}$ basic fibroblast growth factor (bFGF), $10 \mu \mathrm{g} / \mathrm{L}$ epidermal growth factor (EGF), $10^{5} \mathrm{U} / \mathrm{L}$ penicillin, and $100 \mathrm{mg} / \mathrm{L}$ streptomycin.

2.2. Induced Differentiation of Human Pancreatic Progenitor Cells. The expansion induction of human fetal pancreatic progenitor cells was as described previously [3]. Thus, human pancreatic progenitor cells at the 10th gestational week were induced in M199 medium containing 15\% fetal bovine serum, $10 \mathrm{mmol} / \mathrm{L}$ nicotinamide, $30 \mathrm{ng} / \mathrm{mL}$ all-trans retinoic acid, and $42 \mathrm{ng} / \mathrm{ml}$ glucagon-like peptide- 1 for 1 week. The medium was replaced every three days.

2.3. RNA Isolation and Quantitative Real-Time PCR Analysis. Total RNA from induced hFPPCs was extracted using RNAiso Plus (TaKaRa Biotechnology, Dalian, China). Singlestranded cDNAs were generated with Bestar ${ }^{\mathrm{TM}}$ qPCR RT Kit (DBI Bioscience, Shanghai, China). Real-time PCR was conducted by using Bestar qPCR Mastermix SYBR green (DBI Bioscience, Shanghai, China) in ABI prism 7500 Sequence Detection System. Analysis of relative gene expression was measured by quantitative real-time PCR and the $2^{-\Delta \Delta C T}$ Method. The pancreatic stem cell markers (Oct4, Nanog and CK 19), as well as endocrine and $\beta$ cell markers (Ngn3, insulin, GLUT2, Kir6.2, SUR1, and VDCC), were evaluated during differentiation. The mRNA levels of tested markers were normalized to GAPDH.

2.4. Western Blotting. Cell pellets were incubated in RIPA lysis buffer (Beyotime, Nantong, China) supplemented with $1 \mathrm{mM}$ protease inhibitor cocktail (CALBIOCHEM, USA) for 30 minutes on ice, followed by centrifugation at $12,000 \mathrm{rpm}$ for 10 minutes at $4^{\circ} \mathrm{C}$. Cell lysates were resolved using SDSPAGE gels and transferred onto a polyvinylidene difluoride (PVDF) membrane by electrophoresis. The membranes were immunoblotted with the monoclonal rabbit anti-FoxO1 ( $1: 1000$, Cell Signaling, Danvers, MA, USA); the monoclonal mouse anti- $\beta$-actin $(1: 2000$, Santa Cruz Biotechnology, Inc., Santa Cruz, CA, USA), followed by incubation with a goat anti-rabbit secondary antibody $(1: 3000$, Santa Cruz Biotechnology, Inc., Santa Cruz, CA, USA) at room temperature for $2 \mathrm{~h}$. Immunoreactive bands were revealed by enhanced chemiluminescence (SuperSignal ${ }^{\circledR}$ West Pico Chemiluminescent Substrate kits, Thermo Scientific) and visualized by the
KODAK Image Station 4000MM PRO imaging system and software. Band intensities were quantified by scanning densitometry (Gel-Doc2000, Bio-Rad), analyzed with Quantity $\mathrm{One}^{\mathrm{TM}}$ (Bio-Rad), and normalized against the level of $\beta$-actin.

2.5. RNAi Transfection. Transfection of FoxO1-siRNA and control siRNA was according to the typical RNAiMAX transfection procedure. Briefly, siRNA was diluted with OptiMEM Medium into $0.2 \mu \mathrm{m} / \mathrm{L}$ and mixed with diluted Lipofectamine RNAiMAX Reagent. The mixture was incubated for 5 minutes at room temperature and the siRNA-lipid complex was added into cells afterwards.

2.6. Insulin Content Measurement. Human fetal pancreatic progenitor cells transfected with siRNAs were collected and lysed for protein extraction. $25 \mu \mathrm{l}$ supernatant of sample lysate was used for insulin measurement with human insulin ELISA kits (ALPCO Diagnostic, Salem, NH, USA).

2.7. Immunofluorescent Staining. For immunofluorescent staining, human fetal pancreatic progenitor cells transfected with siRNAs were fixed in 4\% paraformaldehyde in PBS for $30 \mathrm{~min}$ at room temperature. Then, they were transferred to membrane permeabilization solution (0.3\% Triton X-100) for $20 \mathrm{~min}$ and blocking buffer (1\% BSA-supplemented PBS) for $1 \mathrm{~h}$. At last, cells were incubated overnight at $4^{\circ} \mathrm{C}$ with antibodies in appropriate dilutions.

2.8. Retroviral Infection. pMX-puro-FoxO1-AAA and control vector for retroviral packaging were cotransfected with psi2 helper plasmid into 293 T cells using the calcium chloride precipitation method. The generated recombinant virus was collected and transfected into hFPPCs, followed by selection in $2 \mu \mathrm{g} / \mathrm{ml}$ puromycin (Sigma) for 4 days.

2.9. Statistical Analyses. Data are presented as mean \pm SEM for the indicated number of experiments $(n)$. Statistical significance was evaluated using the independent $t$-test. Data were considered significant when $p<0.05$.

\section{Results}

3.1. Induction of Human Fetal Pancreatic Progenitor Cells. Human pancreatic progenitor cells derived from 10-week fetal pancreas were induced for differentiation for 7 days as described before [3]. We first examined the expression of the stem cell markers (Oct4 and Nanog) $[12,13]$, pancreatic ductal cell markers (CK19), pancreatic endocrine marker (Ngn3), and the $\beta$ cell marker (insulin) in human pancreatic progenitor cells before and after 7-day induction. qRT-PCR analyses revealed that mRNA levels of Oct4, Nanog, and CK19 were decreased upon induction. By contrast, levels of Ngn3 and insulin designated for endocrine and pancreatic $\beta$ cells were significantly increased (Figures $1(\mathrm{a})$ and $1(\mathrm{~b})$ ), which were consistent to the observations made in the same in vitro induction of the human fetal pancreatic progenitor cells [3].

3.2. Characterization of FoxO1 Expression in the Development of Human Fetal Pancreatic Progenitor Cells. The temporal profiles of FoxO1 were analyzed before and after 7-day 


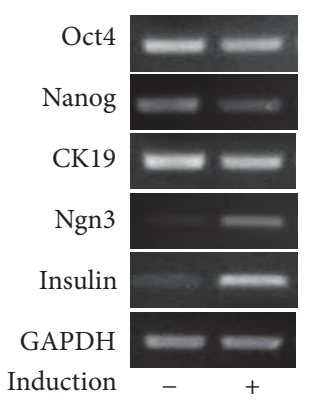

(a)

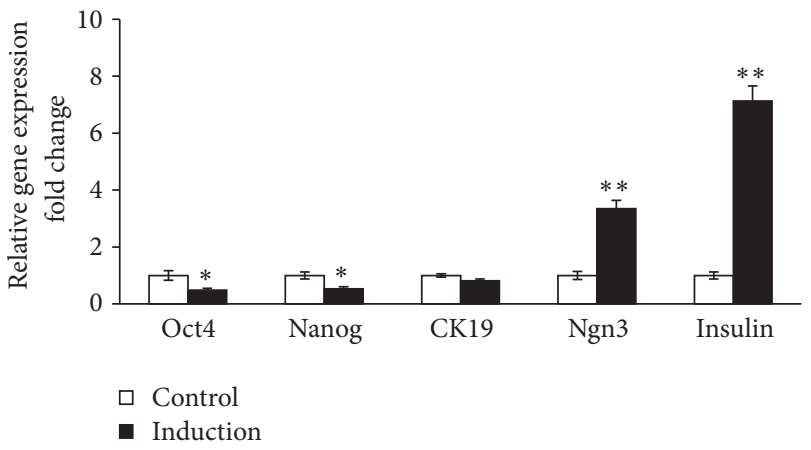

(b)

FIGURE 1: Induction of human pancreatic progenitor cells. (a) Human fetal pancreatic progenitor cells of 10 week were cultured and induced for 1 week. RT-PCR analysis was used to evaluate the markers for stem cell and pancreatic $\beta$ cell in cells before and after 1-week induction. GADPH was used as internal control. (b) Values were expressed as percentage of mRNA expression in cells of induction before (empty bars) and 1-week induction (black bars). Data are means \pm SEM of 4 independent experiments per each group. $*$ or $* *, p<0.05$ or 0.01 .

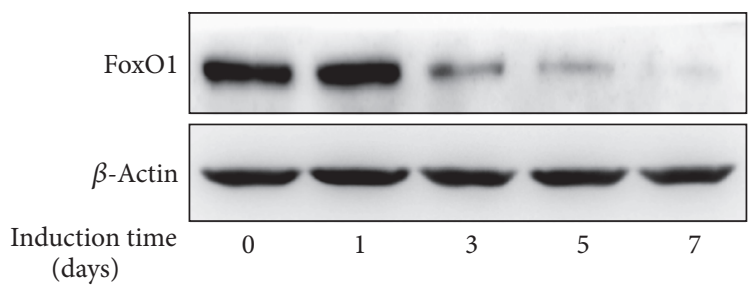

(a)

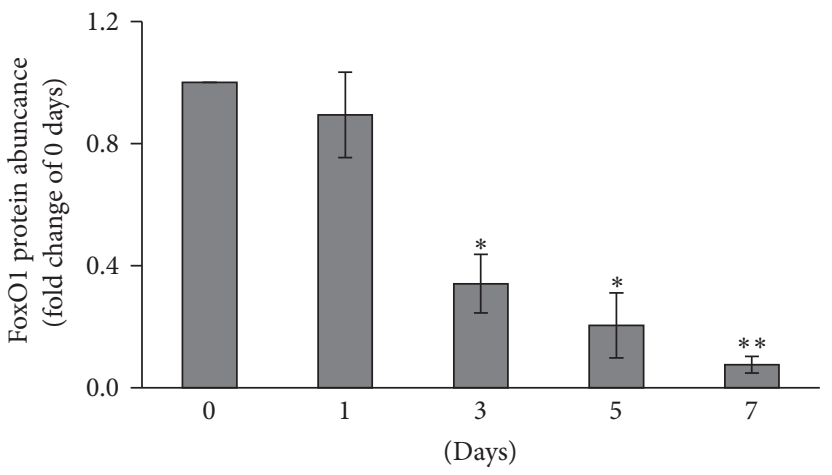

(b)

FIGURE 2: Reduced FoxO1 protein level during induction of human pancreatic progenitor cells. (a) Time course analysis of FoxO1 expression in human pancreatic progenitor cells after induction of differentiation for the time as indicated. Expression of FoxO1 protein was determined by Western blotting. $\beta$-Actin was used as loading control. (b) Data were normalized to the level of $\beta$-actin and expressed as fold change relative to control. Values are means \pm SEM of 3 independent experiments per each group. $*$ or $* *, p<0.05$ or 0.01 .

induction of human fetal pancreatic progenitor cells by Western blotting. As shown in Figure 2(a), FoxO1 protein level decreased in a time-dependent manner during 7-day induction. Indeed, FoxO1 protein level at the 7 th day was $7.5 \pm 2.6 \%(p<0.01)$ of that at control (before induction) (Figure 2(b)).

\subsection{Knockdown of FoxO1 Promotes Cell Differentiation in} Human Fetal Pancreatic Progenitor Cells. We next determined the role of FoxO1 in the induction of human fetal pancreatic progenitor cells. The experiments were performed by transfection of FoxO1 siRNA in the human pancreatic progenitor cells for $24 \mathrm{~h}$. RNAi transfection resulted in $\sim 65 \%$ reduction of FoxO1 level in human fetal pancreatic progenitor cells (Figure 3(a)). This is accompanied by significant increase of Ngn3, insulin, Glut2, Kir6.2, SUR1, and VDCC, as compared to cells transfected with control siRNA (Figure 3(b)). Consistent with the findings by the qRT-PCR analysis, progenitor cells transfected with FoxO1 siRNA showed substantial increase in insulin content (Figure 3(c)), as well as insulin immunoreactivity (Figure 3(d)). This result was supported by the experiments of treatment of human fetal pancreatic progenitor cells with $0.1 \mu \mathrm{M}$ AS 1842856 , the specific FoxO1 inhibitor [14] for 6 days. In this series of experiments, treatment of FoxO1 inhibitor resulted in $\sim 2$ - to $\sim 6$-fold ( $p<$ 0.05 or 0.01 ) increase of mRNA levels for Ngn3, insulin, Glut2, SUR1, and VDCC, respectively (Figure 4).

\subsection{Constitutive Activation of FoxO1 Inhibits Differentiation of} Human Pancreatic Progenitor Cells. To confirm the importance of FoxO1 for cell differentiation, we employed gainof-function approach by transfection of FoxOl-AAA overexpression plasmid (residues Thr24, Ser256, and Ser319 are mutated to Ala) in human fetal pancreatic progenitor cells. In this case, activated FoxO1 would be constantly expressed in the cells. Quantitative analysis revealed that FoxO1-AAA transfected cells exhibited $\sim 8$-fold $( \pm 0.53 ; p<0.01)$ increase of FoxO1 mRNA level, as compared to cells transfected with vector control (Figure 5(a)). Next, we examined the effect of FoxO1 overexpression on cell differentiation in human 


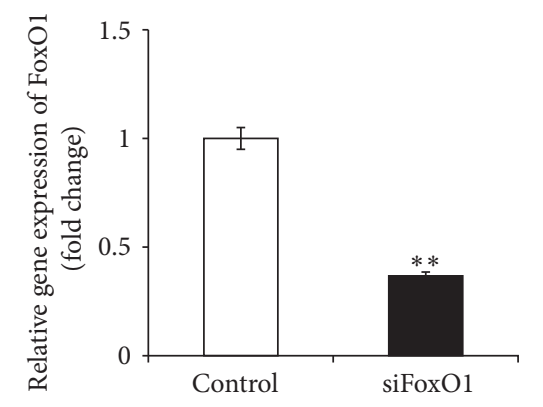

(a)

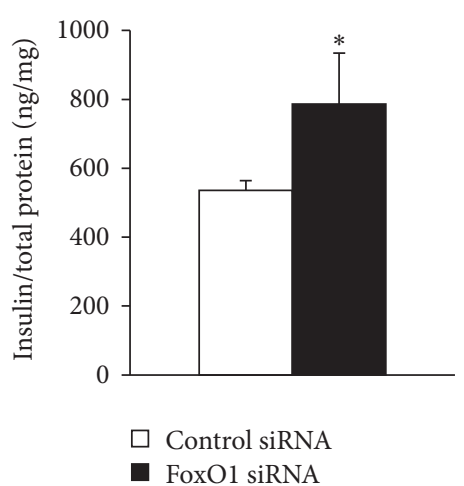

(c)

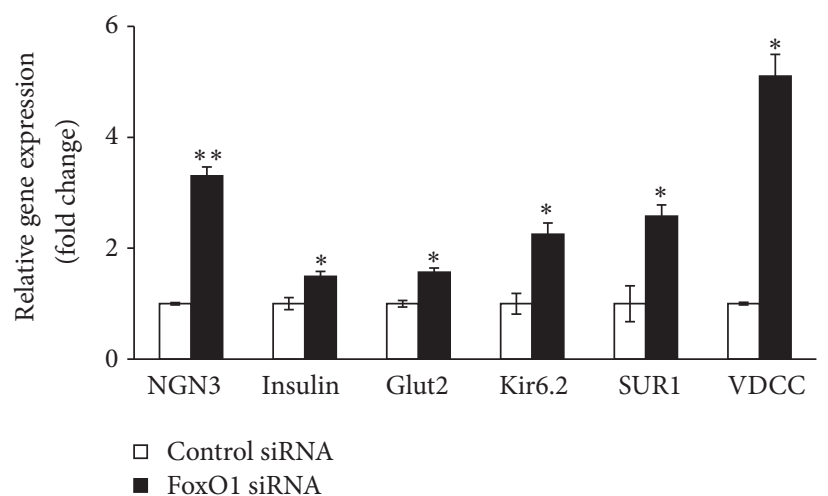

(b)

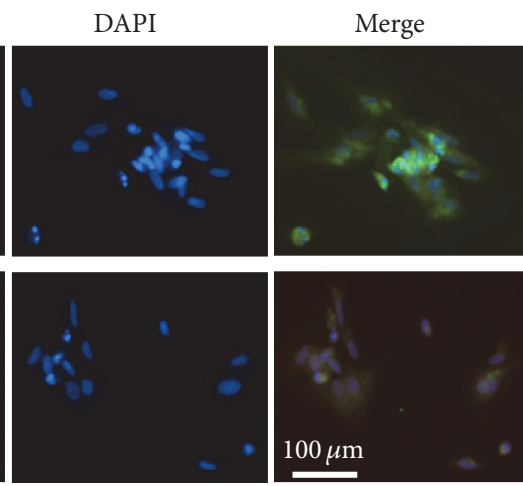

(d)

FIGURE 3: Knockdown of FoxO1 results in increased expression of $\beta$ cell markers. (a) qRT-PCR analysis of FoxO1 mRNA levels in human pancreatic progenitor cells transfected with scramble or FoxO1 siRNA for $48 \mathrm{~h}$. Data were normalized against the level of $\beta$-actin and expressed as fold change relative to scramble siRNA group. Values are means \pm SEM of 4 independent experiments per each group. ${ }^{* *} p<0.01$. (b) qRT-PCR analysis of $\beta$ cell markers in human pancreatic progenitor cells transfected with scramble or FoxO1 siRNA for $48 \mathrm{~h}$. Data were expressed as fold change relative to scramble siRNA group and are means \pm SEM of 4 to 5 independent experiments. $*$ or $* *, p<0.05$ or 0.01. (c) Insulin content analysis by ELISA for human fetal progenitor cells, respectively, transfected with FoxO1 siRNA or control siRNA. Values were normalized to total protein content. ${ }^{*} p<0.05$. (d) Immunohistochemistry with antibodies against FoxO1 (green, dilution of $1: 100$ ) and insulin (red, dilution of 1:100) in the human fetal progenitor cells, respectively, transfected with FoxO1 siRNA (under panels) or control siRNA (upper panels). Arrows indicate the FoxO1 knockdown cells where the insulin was also decreased. Bar $=100$ um.

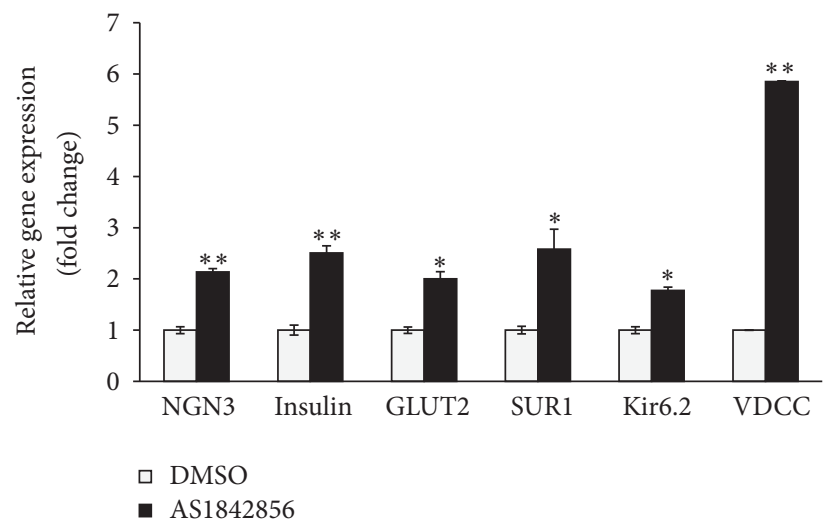

FIGURE 4: FoxO1 inhibitor increased mRNA expression of $\beta$ cell markers. Human fetal pancreatic progenitor cells were treated with DMSO or AS1842856 for 3 days. qRT-PCR analysis of $\beta$ cell markers. Data were expressed as fold change relative to DMSO group and are means \pm SEM of 4 independent experiments per each group. $*$ or $* *, p<0.05$ or 0.01 . 


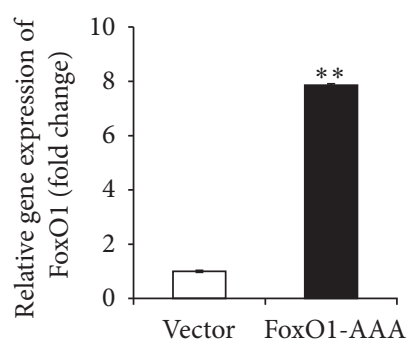

(a)

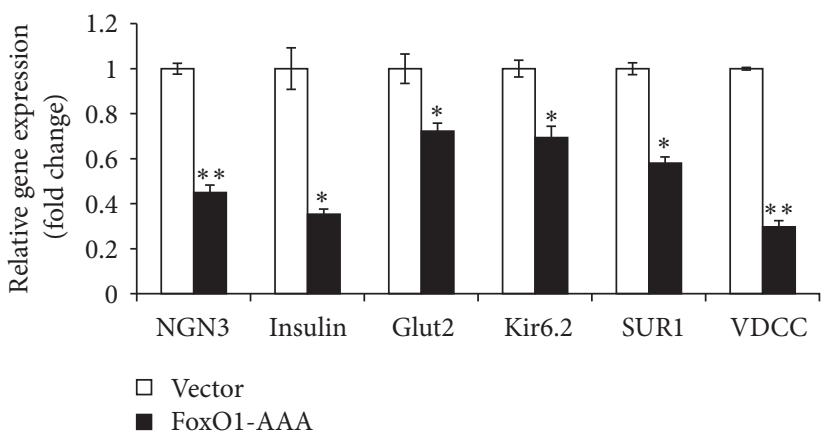

(b)

Figure 5: Constitutive activation of FoxO1 results in decrease of $\beta$ cell markers. (a) qRT-PCR analysis of FoxO1 mRNA levels in human pancreatic progenitor cells transfected with retrovirus expressing either vector or constitutively active FoxO1 (FoxO1-AAA). Data were normalized against the level of GAPDH and expressed as fold change relative to vector (low panel). Values are means \pm SEM of 4 independent experiments per each group. ${ }^{* *} p<0.01$. (b) qRT-PCR analysis of $\beta$ cell markers in human pancreatic progenitor cells transfected with retrovirus expressing either vector or constitutively active FoxO1 (FoxO1-AAA). Data were expressed as fold change relative to vector and are means \pm SEM of 3 to 5 independent experiments per each group. $*$ or $* *, p<0.05$ or 0.01 .

fetal pancreatic progenitor cells. As shown in Figure 5(b), overexpression of FoxO1 in human pancreatic progenitor cells resulted in $\sim 30 \%$ to $\sim 70 \%$ ( $p<0.05$ or 0.01 ) reduction of mRNA levels of Ngn3, insulin, Glut2, Kir6.2, SUR1, and VDCC, respectively (Figure 5(b)). Thus, FoxO1 played a negative role in cell differentiation of human fetal pancreatic progenitor cells.

\section{Discussion}

It has been established that deficient of $\beta$ cell mass plays a vital role in pathogenic process of type 2 diabetes mellitus. Islet transplantation has been suggested as an effective therapeutic strategy to replenish $\beta$ cell mass in both diabetic animals and subjects [15]. Unfortunately, its application is limited by the shortage of available islet supplies. Human fetal pancreatic progenitor cells could be a potential good source of insulin producing cells, as it has a better self-renewal capacity and readily differentiates into insulin producing cells [16]. Our colleagues reported previously that human fetal pancreatic progenitor cells were readily induced into insulin producing cells with higher insulin content and glucose responsiveness, upon in vitro expanded and differentiated in medium for 4 weeks [3]. However, the molecular of control differentiation is still not known. In this study, we got same differentiated human fetal pancreatic progenitor cells at the 10th gestational week by using the similar approach of induction as reported by Zhang et al. [3]. To analyze the role of FoxO1 in the early stage (8-12 weeks) in human fetal pancreatic development [17], we induced the human fetal pancreatic progenitor cells in vitro for 1 week in our study, which correspond to the 12 week fetal development. Notably, we demonstrate that the transcription factor FoxO1 is present in the human fetal pancreatic progenitor cells and acts as a repressor for cell differentiation during the early fetal pancreatic development.

As a transcription factor, the forkhead transcription factor $\mathrm{FoxO}$ is known to involve in various biological process, owing to its ability to bind to conserved DNA sequence $5^{\prime}$-TTGTTTAC- $3^{\prime}$ [18], thus transcriptionally activating or inhibiting a series of downstream targets. It has been found that FoxO1 preferentially expresses in the adult pancreatic $\beta$ cells [6] and plays a critical role in $\beta$ cell growth [5]. Our present study suggested that FoxO1 was also expressed in human fetal pancreatic progenitor cells (Figure 2), which is in agreement with the report by Al-Masri et al. [17], who found that FoxO1 were widely produced during human fetal endocrine pancreatic development. Thus FoxOl may be implicated in regulation of $\beta$ cell differentiation in human fetal pancreatic progenitor cells. Three pieces of evidence corroborate this notion. First, FoxO1 was abundantly expressed in human fetal pancreatic progenitor cells at the beginning of induction, whereas FoxO1 level decreased throughout 7day induction (Figures 2(a) and 2(b)). Second, knockdown (Figure 3) or inhibition (Figure 4) of FoxO1 resulted in significant increase of Ngn3, a critical transcription factor in controlling $\beta$ cell differentiation. Consistently, FoxO1 was found to colocalize with the transcription factor Ngn3 during human fetal endocrine pancreatic development [17]. Increased expression of Ngn3 is accompanied by significant increase of insulin, Glut2, Kir6.2, SUR1, and VDCC, which are essential for mature and function of $\beta$ cells. Third, transfection of human fetal pancreatic progenitor cells with constitutive active FoxO1 resulted in reduced levels of Ngn3, insulin, Glut2, Kir6.2, SUR1, and VDCC (Figure 5).

\section{Conclusion}

In summary, our results indicate the expression and potential function of FoxO1 in the development of human fetal pancreatic progenitor cells. Its inhibitory effects on transcription factors critical for $\beta$ cell differentiation suggest that FoxO1 could be a molecular target for generating insulin producing cells.

\section{Competing Interests}

The authors do not have any potential conflict of interests associated with this research. 


\section{Authors' Contributions}

Zongzhe Jiang and Jingjing Tian contributed equally to this work.

\section{Acknowledgments}

This work was supported by the National Basic Research Program of China (no. 2012CB966402); the Natural Science Foundation of China (no. 81600597); the Strategic Funds for Scientific and Innovative Development of Shenzhen Municipality (no. CXZZ20130329101949981 and no. JSGG20130918150446437); the Peacock Program of Shenzhen Municipality (no. KQTD20140630100746562; no. KQC201108300039A).

\section{References}

[1] J. A. Fridell, J. Rogers, and R. J. Stratta, "The pancreas allograft donor: current status, controversies, and challenges for the future," Clinical Transplantation, vol. 24, no. 4, pp. 433-449, 2010.

[2] Z.-X. Yao, M.-L. Qin, J.-J. Liu, X.-S. Chen, and D.-S. Zhou, "In vitro cultivation of human fetal pancreatic ductal stem cells and their differentiation into insulin-producing cells," World Journal of Gastroenterology, vol. 10, no. 10, pp. 1452-1456, 2004.

[3] W.-J. Zhang, S.-Q. Xu, H.-Q. Cai et al., "Evaluation of islets derived from human fetal pancreatic progenitor cells in diabetes treatment," Stem Cell Research and Therapy, vol. 4, no. 6, article 141, 2013.

[4] S. C. Talchai and D. Accili, "Legacy effect of foxol in pancreatic endocrine progenitors on adult $\beta$-cell mass and function," Diabetes, vol. 64, no. 8, pp. 2868-2879, 2015.

[5] T. Kitamura, J. Nakae, Y. Kitamura et al., "The forkhead transcription factor Foxol links insulin signaling to $P d x$ lregulation of pancreatic $\beta$ cell growth," Journal of Clinical Investigation, vol. 110, no. 12, pp. 1839-1847, 2002.

[6] J. Buteau and D. Accili, "Regulation of pancreatic $\beta$-cell function by the forkhead protein FoxO1," Diabetes, Obesity and Metabolism, vol. 9, no. 2, pp. 140-146, 2007.

[7] T. Kitamura and Y. I. Kitamura, "Role of FoxO proteins in pancreatic $\beta$ cells," Endocrine Journal, vol. 54, no. 4, pp. 507-515, 2007.

[8] T. Kitamura, Y. I. Kitamura, M. Kobayashi et al., "Regulation of pancreatic juxtaductal endocrine cell formation by FoxO1," Molecular and Cellular Biology, vol. 29, no. 16, pp. 4417-4430, 2009.

[9] C. Talchai, S. Xuan, T. Kitamura, R. A. DePinho, and D. Accili, "Generation of functional insulin-producing cells in the gut by Foxol ablation," Nature Genetics, vol. 44, no. 4, pp. 406-412, 2012.

[10] J. Nakae, W. H. Biggs III, T. Kitamura et al., "Regulation of insulin action and pancreatic $\beta$-cell function by mutated alleles of the gene encoding forkhead transcription factor FoxO1," Nature Genetics, vol. 32, no. 2, pp. 245-253, 2002.

[11] H.-J. Kim, M. Kobayashi, T. Sasaki et al., "Overexpression of FoxO1 in the hypothalamus and pancreas causes obesity and glucose intolerance," Endocrinology, vol. 153, no. 2, pp. 659-671, 2012.
[12] H. Niwa, J.-I. Miyazaki, and A. G. Smith, "Quantitative expression of Oct-3/4 defines differentiation, dedifferentiation or selfrenewal of ES cells," Nature Genetics, vol. 24, no. 4, pp. 372-376, 2000.

[13] K. Mitsui, Y. Tokuzawa, H. Itoh et al., “The homeoprotein nanog is required for maintenance of pluripotency in mouse epiblast and ES cells," Cell, vol. 113, no. 5, pp. 631-642, 2003.

[14] T. Nagashima, N. Shigematsu, R. Maruki et al., "Discovery of novel forkhead box $\mathrm{O} 1$ inhibitors for treating type 2 diabetes: improvement of fasting glycemia in diabetic $\mathrm{db} / \mathrm{db}$ mice," Molecular Pharmacology, vol. 78, no. 5, pp. 961-970, 2010.

[15] N. Kobayashi, "The current status of islet transplantation and its perspectives," Review of Diabetic Studies, vol. 5, no. 3, pp. 136143, 2008.

[16] M. V. Joglekar, V. M. Joglekar, S. V. Joglekar, and A. A. Hardikar, "Human fetal pancreatic insulin-producing cells proliferate in vitro," Journal of Endocrinology, vol. 201, no. 1, pp. 27-36, 2009.

[17] M. Al-Masri, M. Krishnamurthy, J. Li et al., "Effect of forkhead box O1 (FOXO1) on beta cell development in the human fetal pancreas," Diabetologia, vol. 53, no. 4, pp. 699-711, 2010.

[18] T. Furuyama, T. Nakazawa, I. Nakano, and N. Mori, "Identification of the differential distribution patterns of mRNAs and consensus binding sequences for mouse DAF-16 homologues," Biochemical Journal, vol. 349, no. 2, pp. 629-634, 2000. 


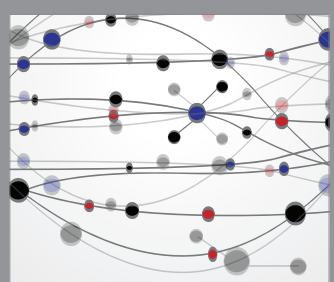

The Scientific World Journal
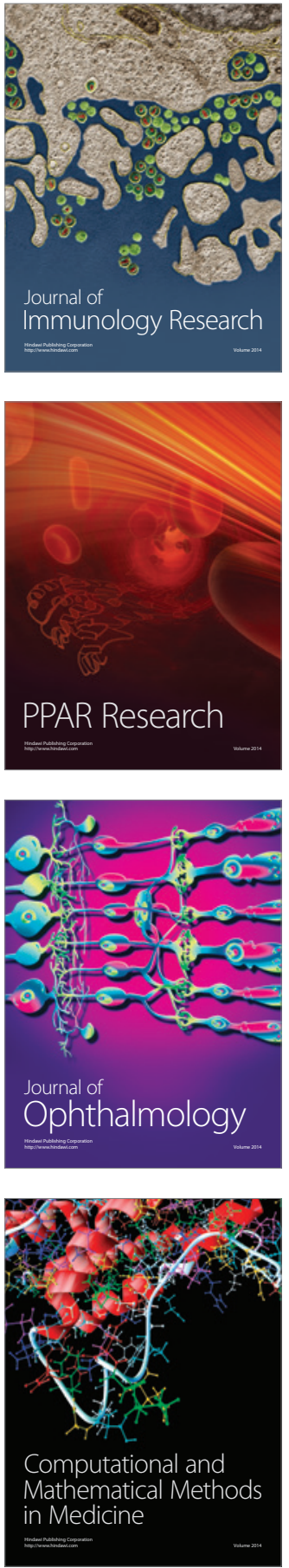

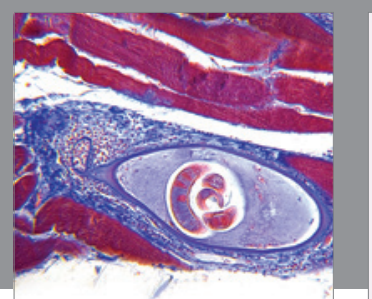

Gastroenterology Research and Practice
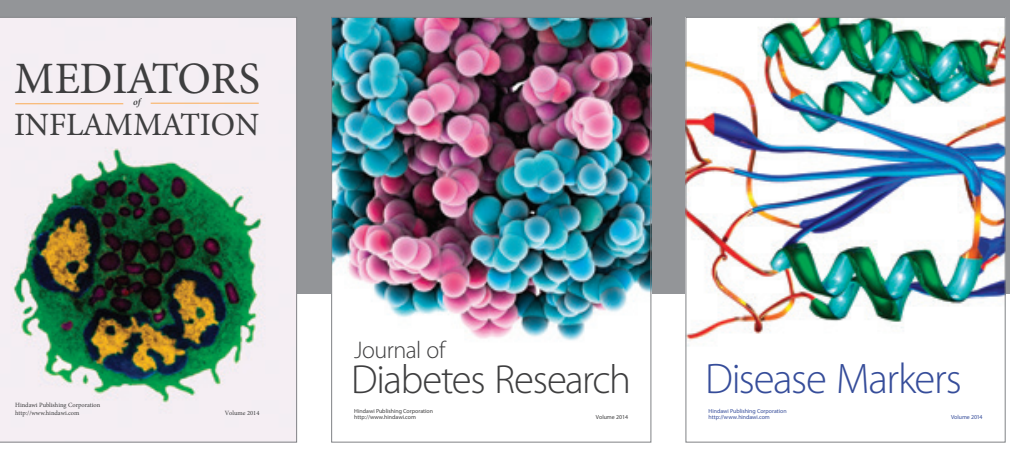

Disease Markers

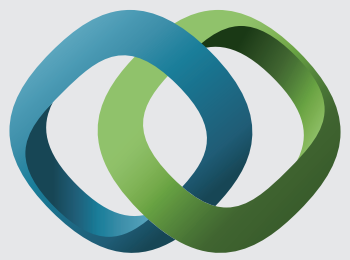

\section{Hindawi}

Submit your manuscripts at

https://www.hindawi.com
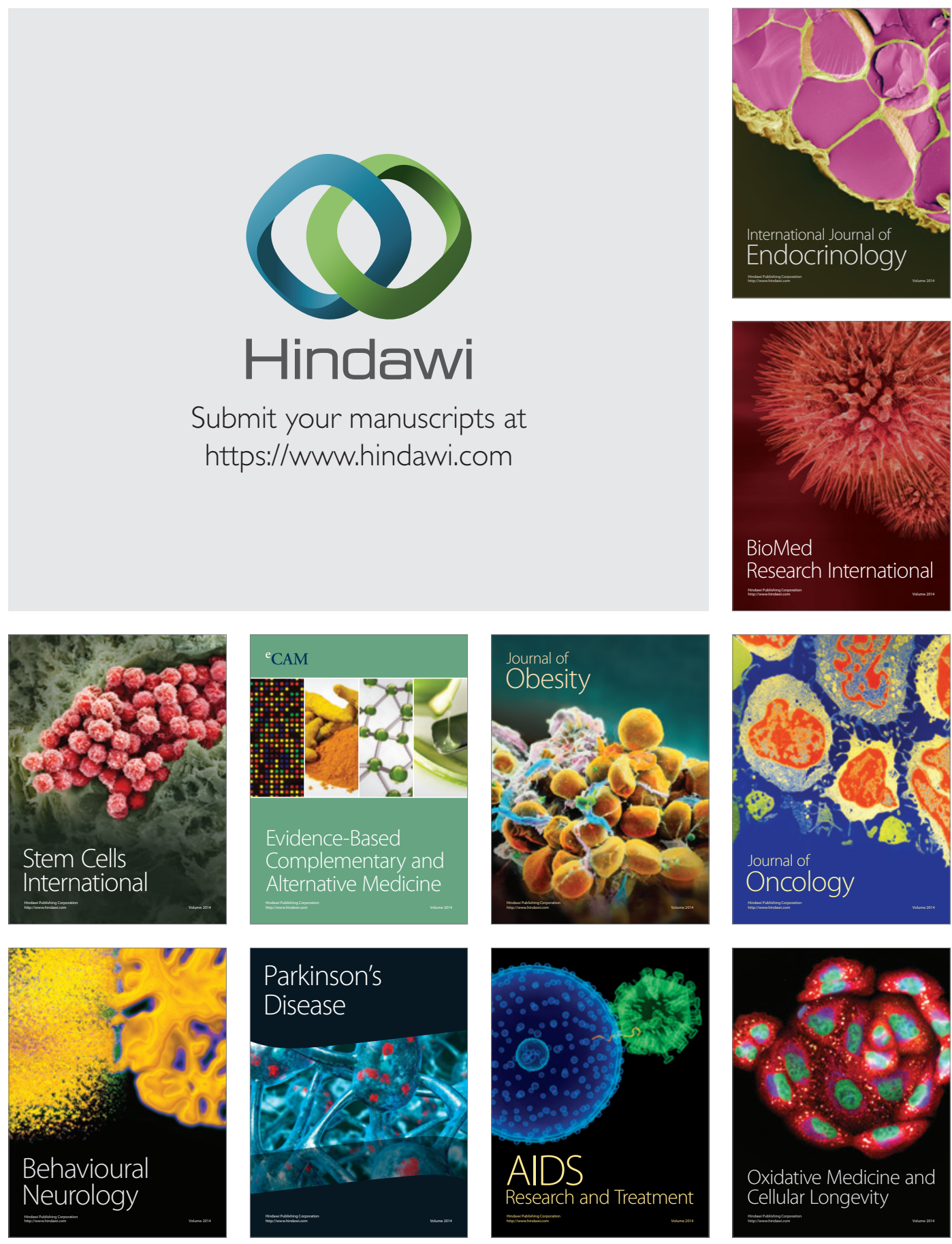